\title{
The effect and safety of preoperative biliary drainage in patients with hilar cholangiocarcinoma: an updated meta- analysis
}

Fei Teng, You-yin Tang, Jun-long Dai, Yongkun Li and Zhe-yu Chen º

\begin{abstract}
Background: The effect and safety of preoperative biliary drainage (PBD) in patients with perihilar cholangiocarcinoma are still controversial; the aim of our study is to evaluate all aspects of PBD.

Methods: All included studies featured PBD versus non-PBD (NPBD) groups were from 1996 to 2019 and were extracted from Cochrane Library, Embase, PubMed, and Science Citation Index Expanded.

Results: Sixteen studies met the inclusion criteria and were included in this analysis. PBD may lead to a significantly higher incidence of overall morbidities $(\mathrm{OR} 0.67,95 \% \mathrm{Cl} 0.53,0.85 ; P=0.0009)$ and intraoperative transfusions (OR $0.72,95 \% \mathrm{Cl} 0.55,0.94 ; P=0.02)$; moreover, bile leakage (OR 0.58, 95\% Cl 0.24, 1.41; $P=0.04$ ), infection (OR 0.31, $95 \% \mathrm{Cl} 0.20,0.47 ; P<0.00001)$, and cholangitis (OR $0.18,95 \% \mathrm{Cl} 0.007,0.48 ; P=0.0007)$ are also related to PBD. However, NPBD was associated with more frequent hepatic insufficiency $(\mathrm{OR} 3.09,95 \% \mathrm{Cl} 1.15,8.31 ; P=0.03)$. In the subgroup meta-analysis, the differences in the outcomes of bile leakage and overall morbidity lost significance between the PBD and NPBD groups when the mean total serum bilirubin (TSB) concentration was above $15 \mathrm{mg} / \mathrm{dl}$.

Conclusion: Meta-analysis demonstrated that compared to NPBD, PBD is associated with a greater risk of several kinds of infection and morbidities, but its ability to reduce postoperative hepatic insufficiency cannot be ignored. In patients with a high TSB concentration, PBD tends to be a better choice. However, these results need to be confirmed in a future prospective randomized trial with large samples to clarify the effects and find a specific TSB concentration for PBD.
\end{abstract}

Keywords: Hilar cholangiocarcinoma, Klatskin tumor, Preoperative biliary drainage, Meta-analysis, Total serum bilirubin

\section{Introduction}

Hilar cholangiocarcinoma (hCCA), which is also known as a Klatskin tumor, is the most prevalent type of all carcinomas related to bile ducts (accounting for 50-60\%) [1-3]. hCCA that arises in the proximal extrahepatic epithelium of the bile ducts involving the right and left

* Correspondence: chenzheyu@scu.edu.cn

Department of Liver Surgery, Liver Transplantation Center, West China Hospital of Sichuan University, Chengdu 610041, Sichuan Province, China main hepatic ducts is defined as cholangiocarcinoma. Currently, the only curative treatment for hCCA is surgery that includes extrahepatic bile duct resection, probably with major hepatic resection and potential hepatoduodenal lymphadenectomy [1-4]. However, the majority of patients with hCCA have no typical symptoms until hCCA progresses enough to obstruct the bile ducts and lead to jaundice, which always presents as the first symptom. Unfortunately, according to several

C C The Author(s). 2020 Open Access This article is licensed under a Creative Commons Attribution 4.0 International License, which permits use, sharing, adaptation, distribution and reproduction in any medium or format, as long as you give appropriate credit to the original author(s) and the source, provide a link to the Creative Commons licence, and indicate if changes were made. The images or other third party material in this article are included in the article's Creative Commons licence, unless indicated otherwise in a credit line to the material. If material is not included in the article's Creative Commons licence and your intended use is not permitted by statutory regulation or exceeds the permitted use, you will need to obtain permission directly from the copyright holder. To view a copy of this licence, visit http://creativecommons.org/licenses/by/4.0/. The Creative Commons Public Domain Dedication waiver (http://creativecommons.org/publicdomain/zero/1.0/) applies to the data made available in this article, unless otherwise stated in a credit line to the data. 
studies, patients with jaundice may experience infections, postoperative hepatic insufficiency, intraoperative blood loss, and renal insufficiency [4-8], and preoperative biliary drainage (PBD) is regarded as a practical solution to reduce the total serum bilirubin (TSB) concentration. However, patients who undergo PBD, mainly by percutaneous transhepatic biliary drainage (PTBD) and endoscopic nasobiliary drainage (ENBD), also have an increased risk for tumor seeding, extended hospital stays, morbidities, and infections $[1,2,9,10]$. Since a balance is needed between benefits and risks, the indications for PBD are still under debate.

In view of this debate, Celotti et al. [11] previously collected nine studies to conduct a meta-analysis of eight kinds of morbidities. However, the number of studies included was small, and several studies that can meet the inclusion criterion have been published in recent years. Moreover, the debate between $\mathrm{PBD}$ versus non-PBD (NPBD) for different TSB concentrations also needs to be clarified with a subgroup analysis. Thus, we conducted a systematic review and meta-analysis to fully evaluate the safety and effect of PBD in patients with hCCA.

\section{Materials and methods}

\section{Literature search}

The work has been reported in line with the Preferred Reporting Items for Systematic Reviews and Metaanalyses (PRISMA) and Assessing the Methodological Quality of Systematic Reviews (AMSTAR) guidelines. A systematic literature search was conducted in the Cochrane Library, Embase, PubMed, and Science Citation Index Expanded to identify and retrieve studies published from January 1995 to December 2019 related to the evaluation of PBD in treating hCCA patients. The following concrete search method was used: Search $(((\mathrm{PBD}[$ Title/Abstract $]) \quad$ OR drainage[Title/Abstract])) AND (((bile duct cancer[Title/Abstract]) OR "Cholangiocarcinoma*[Title/Abstract]) OR "Klatskin"[Title/Abstract]). The language of the articles was limited in English. The references of each selected study were screened for any relevant articles that could be included.

\section{Study selection}

We set the following inclusion and exclusion criteria for the literature we found. The inclusion criteria were as follows: (1) published in English, (2) was human research, (3) included patients with hilar cholangiocarcinoma, and (4) compared PBD versus NPBD; if the same institution (and authors) published multiple studies, the study with either a higher quality or larger sample size was included. Studies were excluded based on the following criteria: (1) letters, editorials, expert opinions, abstracts, and case reports; and (2) duplicate patient populations.

\section{Qualitative assessment of the studies selected}

The risk of bias in the included non-randomized studies was evaluated according to the risk of bias in nonrandomized studies of interventions (ROBINS-1) tool [12].

\section{Data extraction and synthesis}

Each study was screened and evaluated by two investigators independently (Fei Teng and You-yin Tang) for a decision regarding exclusion from the review. If disagreements between the reviewers occurred, a third reviewer (Yongkun $\mathrm{Li}$ ) was consulted. Two investigators separately collected data from every study included with standardized forms. The patients' basic characteristics, quality assessments, intraoperative outcomes, and postoperative outcomes were included. The means and standard deviations were used for continuous variable meta-analysis unless otherwise mentioned. If the means and standard deviations were impossible to access with the median, range, and large sample size provided, we used Hozo's method [13] to approximately estimate the mean and standard deviation.

We extracted the following data from each study: author, year, country, study duration, study design, number of patients in the PBD and NPBD groups, age, sex, body mass index (BMI), TSB before drainage in the PBD groups, preoperative TSB in the NPBD groups, Bismuth classification of the PBD and NPBD groups, mortality, morbidity, hepatic insufficiency, renal insufficiency, R0 resection, operation time ( $\mathrm{min}$ ), need for intraoperative transfusion, and incidence of bile leakage, infection, cholangitis, intra-abdominal abscess, abdominal collection, anastomotic leakage, and second laparotomy.

\section{Outcomes of interest and definitions}

PBD was defined as an approach to reduce serum bilirubin levels before the operation by PTBD or ENBD. The primary outcomes were mortality, which was defined as death occurring within 90 days from admission to the hospital, and morbidity, which was defined as any complication that occurred during hospitalization or within 90 days after surgery. The secondary outcomes included hepatic insufficiency, renal insufficiency, R0 resection, operation time ( $\mathrm{min}$ ), intraoperative transfusion, bile leakage, infection, cholangitis, intra-abdominal abscess, anastomotic leakage, and second laparotomy. Hepatic insufficiency was defined by a standard definition [14]. Renal insufficiency was defined as any decrease in glomerular filtration rate with no reversion to the preoperative level. R0 resection was defined as curative treatment when the resection margin was free of tumor cells according to 
microscopy. Operation time was defined as the interval from incision to suturing of the skin. Intraoperative transfusion was defined as a blood transfusion of at least 1 unit during the operation. Blood loss was defined as aby blood loss during surgery. Infectious complications were defined according to the study by Hochwald et al. [15] and included infection, intraabdominal abscess, and cholangitis. Bile leakage was defined as at least $50 \mathrm{ml}$ of bile drained from the surgical drainage tube or from the drainage of an abdominal collection over a period of 3 days or more [16]. Abdominal collection was defined as a collection of fluid in the abdomen after surgery. Anastomotic leakage was defined according to Dindo D's [17] classification of complications. Second laparotomy was defined as an operation for any curative reason after the previous surgical resection.

\section{Statistical analysis}

A meta-analysis was performed using the Review Manager version 5.3 software (The Cochrane Collaboration, Oxford, UK). For each outcome, $P<0.05$ was considered statistically significant. Continuous variables are expressed as weighted mean differences (WMDs) and odds ratios (ORs) for comparisons, and their corresponding 95\% confidence intervals (CIs) are reported. Categorical variables are reported as ORs with their corresponding 95\% confidence intervals (CIs). A chi-square test was used to evaluate heterogeneity, with $P<0.1$ considered significant. The $I^{2}$ value was used to evaluate statistical heterogeneity, and a value of $50 \%$ or more indicated the presence of heterogeneity [18]. The fixedeffects model was preferred for all outcomes, but if the test rejected the assumption of homogeneity $\left(I^{2}>50 \%\right.$ and heterogeneity $P>0.05)$, we chose the randomeffects model. We also performed a sensitivity analysis for every single study included to determine the source of heterogeneity. For every outcome we measured, we performed a funnel plot to evaluate potential publication bias.

\section{Results}

\section{Description of the included studies}

We reported this systematic review in accordance with the PRISMA statement [19]. A flow diagram of the search process for studies is shown in Fig. 1. In total, 3625 studies were identified from the electronic databases we mentioned, and 2539 studies were removed due to duplicate publications. Finally, only 40 studies were fully screened for eligibility; however, for many reasons, 23 studies were excluded, and only 17 studies were included in our research. Unfortunately, the study conducted by Figueras et al. [20] was excluded after a discussion among our group because of missing data. Thus,
16 studies with 1860 (NPBD, 775 patients; PBD, 1085 patients) patients were included in the meta-analysis [21-36]. Because all of the included studies were retrospective comparative studies, the bias outcomes we measured with the ROBINS-I tool are shown in Table 1. Some studies were considered to have a serious risk of bias [22, 27, 28, 32, 34, 35]; some studies were considered to have a moderate risk of bias $[25,26,30,31,33$, $36]$, and some studies were considered to have a low risk of bias [21, 23, 24]. The characteristics of the included patients are shown in Table 2. The basic perioperative and hilar cholangiocarcinoma data are shown in Tables 3 and 4 ; the results of the meta-analysis are shown in Table 5; the results of the subgroup meta-analysis are shown in Fig. 2; the publication bias measured by the funnel plot is shown in Fig. 3, and the forest diagrams are updated in supplied materials.

\section{Results of the meta-analysis}

After a common meta-analysis, to evaluate the effects of PBD among different preoperative TSB levels in patients with hCCA, the included studies were divided into two subgroups: TSB $<10 \mathrm{mg} / \mathrm{dl}$ before PBD [21, 23, 25, 26, 30], and TSB > $15 \mathrm{mg} / \mathrm{dl}$ before PBD [24, 29, 31, 33, 36]. However, the outcomes presented only statistically significant differences in morbidity and bile leakage between subgroups.

\section{Primary outcomes}

The mortality rate was reported in all 16 studies [21-36], with no statistically significant difference observed between NPBD and PBD (OR 0.91, 95\% CI 0.64, $1.30 ; P=0.62$ ). The outcome of morbidity was reported in 12 studies [23-25, 27-33, 35, 36], with a significant difference observed between groups; this result showed that PBD may lead to more morbidities (OR 0.67, 95\% CI $0.53,0.85 ; P=0.0009$ ).

\section{Secondary outcomes}

Hepatic insufficiency was reported in 8 studies [22, 24-27, 30, 33, 36], and the NPBD group had a significantly higher risk of suffering from hepatic insufficiency than the PBD group (OR 3.09, 95\% CI 1.15, 8.31; $P=$ $0.03)$. Renal insufficiency was reported in 4 studies [24, $31,33,36]$, and no significant difference was observed between groups (OR 1.07, 95\% CI 0.40, 2.84; $P=0.89$ ). Intraoperative transfusion was reported in 7 studies [21, 23-27, 33], and the PBD group had a significantly higher risk of requiring intraoperative transfusions (OR 0.72, 95\% CI $0.55,0.94 ; P=0.02$ ). The PBD group had a significantly higher incidence of bile leakage according to the outcomes of 6 studies [23, 24, 27, 30,33, 36] (OR $0.58,95 \%$ CI $0.24,1.41 ; P=0.04)$. The proportion of positive surgical margins was reported in 5 studies [21, 


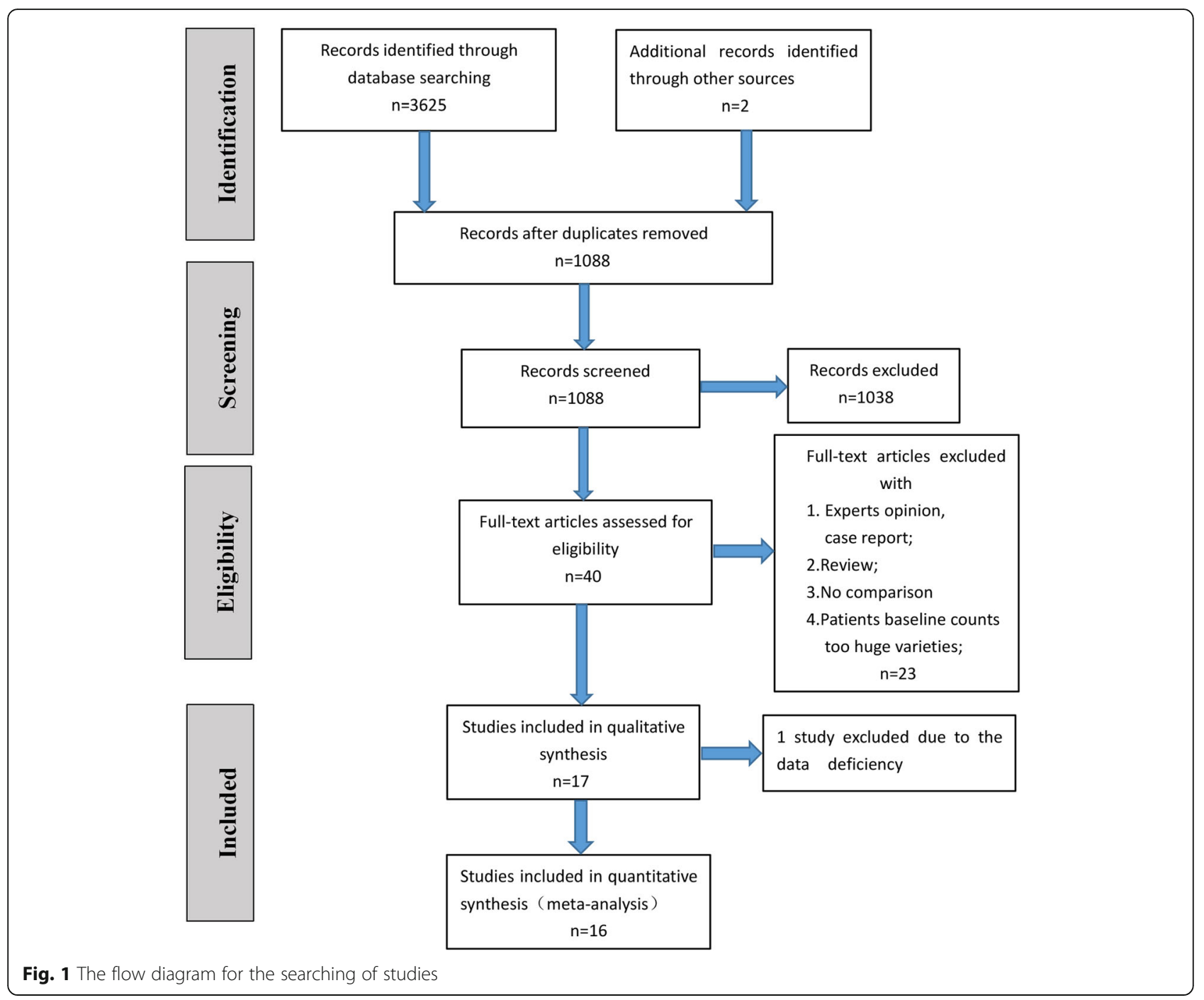

$23,29,30,36]$, and there was no statistically significant difference between groups (OR 1.36, 95\% CI 0.93, 1.96; $P=0.11)$.

Infection was significantly more likely to occur in the PBD group than in the NPBD group, based on the outcomes of 8 studies [23-27, 31, 33, 36] (OR 0.31, 95\% CI $0.20,0.47 ; P<0.00001)$. Cholangitis was reported in only 3 studies [24, 27, 30], and the PBD group had a significantly higher risk of cholangitis (OR 0.18, 95\% CI 0.007, $0.48 ; P=0.0007)$. Intra-abdominal abscess was reported in 5 studies [23-25, 27, 36], without a statistically significant difference between groups (OR 0.48, 95\% CI 0.22, 1.32; $P=0.07$ ). Intraoperative blood loss was reported in 5 studies [21, 23, 24, 30, 36], without a statistically significant difference between groups (WMD 32.34, 95\% CI $375.83,440.51 ; P=0.88)$. The operation time was reported in 5 studies [21, 25, 26, 30,33], without a statistically significant difference between groups (WMD -
63.21, 95\% CI - 156.16, 29.73; $P=0.18$ ). Second laparotomy was reported in 4 studies [21, 24, 26, 27], without a statistically significant difference between groups (OR 1.37, 95\% CI 0.52, 3.63; $P=0.53$ ). Anastomotic leakage was only reported in 3 studies [23, 24, 31], without a statistically significant difference between groups (OR 0.55 , 95\% CI 0.15, 2.10; $P=0.38$ ). Abdominal collection was reported in 3 studies [24, 27, 36], without a statistically significant difference between groups (OR 0.9, 95\% CI $0.4,2.00 ; P=0.79$ ).

\section{Subgroup meta-analysis}

All studies were divided into low TSB concentrations (< $10 \mathrm{mg} / \mathrm{dl})[21,23,25,26,27]$ and high TSB concentrations (>15 mg/dl) [24, 29, 31, 33, 36] according to the mean TSB concentration. All of the primary outcomes and secondary outcomes mentioned were analyzed, but statistically significant differences were only observed in overall 


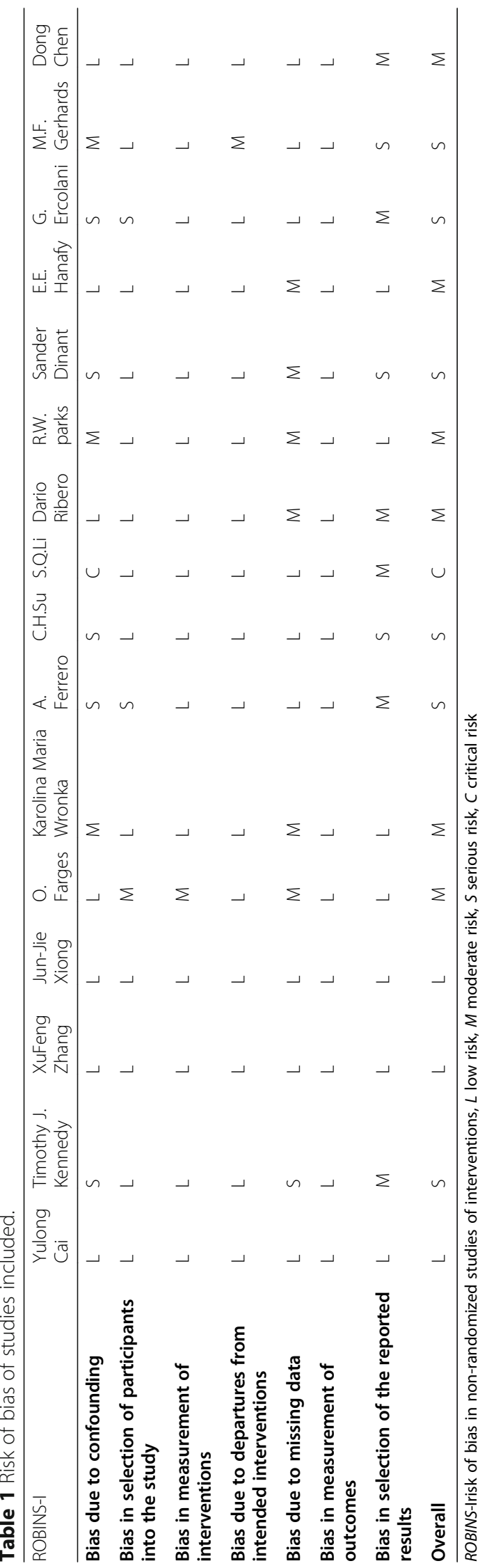


Table 2 Characteristics of the studies included

\begin{tabular}{|c|c|c|c|c|c|c|c|c|c|c|c|c|c|c|c|}
\hline \multirow[t]{2}{*}{ Author } & \multirow[t]{2}{*}{ Country } & \multirow[t]{2}{*}{ Year } & \multirow[t]{2}{*}{ Design } & \multicolumn{2}{|c|}{$\begin{array}{l}\text { No of } \\
\text { patients }\end{array}$} & \multicolumn{2}{|l|}{ Age } & \multicolumn{2}{|l|}{$\mathrm{TSB}(\mu \mathrm{mol} / \mathrm{l})$} & \multicolumn{2}{|c|}{$\operatorname{Sex}(M / F)$} & \multicolumn{2}{|l|}{ BMI } & \multicolumn{2}{|c|}{ Bismuth-classification } \\
\hline & & & & NPBD & PBD & NPBD & PBD & NPBD & PBD & NPBD & PND & NPBD & PBD & NPBD & PBD \\
\hline $\begin{array}{l}\text { Cai et al. } \\
{[21]}\end{array}$ & $\mathrm{CN}$ & 2017 & RETRO & 163 & 55 & $60 \pm 9$ & $59 \pm 11$ & $\begin{array}{l}161.0(8.4- \\
455.6)\end{array}$ & $\begin{array}{l}281.1 \\
(8.2-565.4)\end{array}$ & $74 / 89$ & $\begin{array}{l}30 / \\
25\end{array}$ & $22.5 \pm 6.8$ & $21.8 \pm 5.9$ & $\begin{array}{l}24 / 28 / 24 / \\
42 / 45\end{array}$ & $\begin{array}{l}9 / 13 / 10 / 8 / \\
5\end{array}$ \\
\hline $\begin{array}{l}\text { Kennedy } \\
\text { et al. [22] }\end{array}$ & USA & 2009 & RETRO & 29 & 31 & NR & $N R$ & NR & $N R$ & $N R$ & NR & $N R$ & NR & NR & NR \\
\hline $\begin{array}{l}\text { Zhang } \\
\text { et al. [23] }\end{array}$ & $C N$ & 2017 & RETRO & 44 & 196 & 70 & 67 & $\begin{array}{l}30.8(15.4- \\
99.2)\end{array}$ & $\begin{array}{l}99.2 \\
(39.3-205.2)\end{array}$ & $20 / 22$ & $\begin{array}{l}113 / \\
83\end{array}$ & $\begin{array}{l}25.5(23.7- \\
28.7)\end{array}$ & $\begin{array}{l}25.0(22.3- \\
28.7)\end{array}$ & $\begin{array}{l}15(I-I I) / 37 \\
(I I I-I V)\end{array}$ & $\begin{array}{l}49(I-I I) / 130 \\
(I I I-I V)\end{array}$ \\
\hline $\begin{array}{l}\text { Xiong } \\
\text { et al. [24] }\end{array}$ & $\mathrm{CN}$ & 2013 & RETRO & 46 & 32 & $\begin{array}{l}58.2 \pm \\
11.3\end{array}$ & $\begin{array}{l}59.6 \pm \\
11.0\end{array}$ & $254.0 \pm 63.5$ & $\begin{array}{l}294.2 \pm \\
135.7\end{array}$ & $28 / 18$ & $\begin{array}{l}21 / \\
11\end{array}$ & $21.0 \pm 2.5$ & $20.3 \pm 1.9$ & $\begin{array}{l}1 / 14 / 7 / 15 / \\
9\end{array}$ & $1 / 8 / 6 / 9 / 8$ \\
\hline $\begin{array}{l}\text { Farges } \\
\text { et al. [25] }\end{array}$ & EURO & 2012 & RETRO & 186 & 180 & $62 \pm 11$ & $61 \pm 10$ & $\begin{array}{l}126(17- \\
255)\end{array}$ & $\begin{array}{l}113 \\
(40-274)\end{array}$ & $\begin{array}{l}107 / \\
79\end{array}$ & $\begin{array}{l}127 / \\
53\end{array}$ & $24 \pm 4$ & $25 \pm 4$ & $\begin{array}{l}6 / 47 / 267 / \\
19 / 27\end{array}$ & $\begin{array}{l}4 / 23 / 137 / \\
10 / 6\end{array}$ \\
\hline $\begin{array}{l}\text { Wronka } \\
\text { et al. [26] }\end{array}$ & Poland & 2019 & RETRO & 23 & 58 & $\begin{array}{l}56(48- \\
65)\end{array}$ & $\begin{array}{l}60(55- \\
67)\end{array}$ & $\begin{array}{l}164.8(18.8- \\
336.4)\end{array}$ & $\begin{array}{l}36.6 \\
(15.9-83.8)\end{array}$ & $13 / 10$ & $\begin{array}{l}34 / \\
24\end{array}$ & $N R$ & NR & NR & NR \\
\hline $\begin{array}{l}\text { Ferrero } \\
\text { et al. [27] }\end{array}$ & Italy & 2008 & RETRO & 30 & 30 & $\begin{array}{l}63.5 \\
(35-80)\end{array}$ & $\begin{array}{l}66.0 \\
(47-80)\end{array}$ & $\begin{array}{l}210.3(59.9- \\
533.5)\end{array}$ & 225.7 & $19 / 11$ & $\begin{array}{l}13 / \\
17\end{array}$ & NR & NR & NR & NR \\
\hline $\begin{array}{l}\text { Su et al. } \\
{[28]}\end{array}$ & USA & 1996 & RETRO & 16 & 33 & NR & $N R$ & $N R$ & NR & $N R$ & $N R$ & $N R$ & $N R$ & NR & NR \\
\hline $\begin{array}{l}\text { Li et al. } \\
{[29]}\end{array}$ & $\mathrm{CN}$ & 2009 & RETRO & 56 & 55 & $N R$ & NR & $268 \pm 174$ & $256 \pm 136$ & $N R$ & $N R$ & NR & $N R$ & NR & NR \\
\hline $\begin{array}{l}\text { Ribero } \\
\text { et al. [30] }\end{array}$ & Italy & 2016 & RETRO & 35 & 98 & $\begin{array}{l}67(35- \\
82)\end{array}$ & $\begin{array}{l}65(40- \\
84)\end{array}$ & 94.1 & 63.3 & $22 / 13$ & $\begin{array}{l}62 / \\
36\end{array}$ & $N R$ & NR & $\begin{array}{l}0 / 8 / 12 / 13 / \\
2\end{array}$ & $\begin{array}{l}0 / 17 / 43 / \\
27 / 11\end{array}$ \\
\hline $\begin{array}{l}\text { Parks } \\
\text { et al. [31] }\end{array}$ & UK & 2000 & RETRO & 27 & 20 & $59 \pm 3$ & $57 \pm 2$ & 335 (9-670) & $\begin{array}{l}278 \\
(35-666)\end{array}$ & $20 / 7$ & $16 / 4$ & $N R$ & NR & $2 / 7 / 11 / 5 / 2$ & $2 / 2 / 10 / 2 / 4$ \\
\hline $\begin{array}{l}\text { Dinant } \\
\text { et al. [32] }\end{array}$ & Netherlands & 2006 & RETRO & 14 & 83 & $N R$ & NR & $N R$ & $N R$ & $N R$ & NR & NR & $N R$ & $N R$ & NR \\
\hline $\begin{array}{l}\text { Hanafy } \\
\text { [33] }\end{array}$ & Egypt & 2010 & RETRO & 54 & 46 & $\begin{array}{l}50.4 \pm \\
12\end{array}$ & $53.3 \pm 11$ & $\begin{array}{l}314.6 \pm \\
205.2\end{array}$ & $\begin{array}{l}92.34 \pm \\
126.5\end{array}$ & $34 / 20$ & $\begin{array}{l}30 / \\
16\end{array}$ & NR & NR & $N R$ & NR \\
\hline $\begin{array}{l}\text { Ercolani } \\
\text { et al. [34] }\end{array}$ & Japan & 2010 & RETRO & 7 & 44 & NR & $N R$ & $N R$ & $N R$ & $N R$ & $N R$ & $N R$ & NR & $N R$ & NR \\
\hline $\begin{array}{l}\text { Gerhards } \\
\text { et al. [35] }\end{array}$ & Netherlands & 1999 & RETRO & 18 & 93 & $N R$ & NR & $N R$ & NR & $N R$ & NR & $N R$ & $N R$ & $N R$ & NR \\
\hline $\begin{array}{l}\text { Chen } \\
\text { et al. [36] }\end{array}$ & $C N$ & 2007 & RETRO & 27 & 31 & $N R$ & $N R$ & $382 \pm 174$ & $292 \pm 103$ & $N R$ & NR & NR & NR & $2 / 6 / 3 / 7 / 9$ & $1 / 4 / 6 / 12 / 8$ \\
\hline
\end{tabular}

Data shown represents mean \pm standard deviation or median (minimum-maximum); Bismuth-classification $=1 / \mathrm{ll} / \mathrm{Illa} / \mathrm{Ill} \mathrm{lb} /$

$T S B$ total serum bilirubin, No number, $M$ male, $F$ female, $B M I$ body mass index, $P B D$ preoperative biliary drainage group, $N P B D$ non-preoperative biliary drainage group, Retro retrospective, NR not report

morbidity (low TSB concentration: OR $=0.47,95 \% \mathrm{CI}$ $0.22,1.00, P=0.05$; high TSB concentration: $\mathrm{OR}=0.81$, 95\% CI 0.50, 1.30, $P=0.38$ ) and bile leakage (low TSB concentration: $\mathrm{OR}=0.36,95 \% \mathrm{CI} 0.18,0.72, P=0.004$; high TSB concentration: $\mathrm{OR}=0.92,95 \% \mathrm{CI} 0.27,3.12, P=$ 0.90).

\section{Sensitivity analysis}

We conducted a sensitivity analysis on every study included by changing the type of effects model or excluding individual studies from the outcomes analysis. No results changed when the effect model was simply switched to another model. Although high heterogeneity existed in the outcomes of intraoperative blood loss, hepatic insufficiency, and operation time, the outcomes were stable, with no meaningful or significant changes when the effect model was changed. There was high heterogeneity for liver insufficiency; however, the heterogeneity was zero when Dario's study [30] was excluded.

\section{Discussion}

For several years, jaundice after hepatectomy was regarded as an incident related to hepatic insufficiency or even hepatic failure; moreover, the main reason for death after liver surgery was hepatic failure [37]. In fact, the mechanisms of liver failure caused by hyperbilirubinemia have already been demonstrated in animal experiments, and cholestasis makes the liver more susceptible to ischemia, reperfusion drainage, and inflammation, likely because of a reduction in antioxidant ability and a stronger response to inflammation [38]. However, the routine use of PBD in hCCA is still controversial in clinical practice.

Many experts and researchers from Western countries and Japan suggested the routine use of PBD before surgery in hCCA patients [22, 39-41] due to its 
Table 3 Operative data of primary outcomes and major complications

\begin{tabular}{|c|c|c|c|c|c|c|c|c|c|c|c|c|c|c|}
\hline \multirow{3}{*}{ Author } & \multirow{2}{*}{\multicolumn{2}{|c|}{ Mortality }} & \multirow{2}{*}{\multicolumn{2}{|c|}{ Morbidity }} & \multirow{2}{*}{\multicolumn{2}{|c|}{$\begin{array}{l}\text { Hepatic } \\
\text { insufficiency }\end{array}$}} & \multirow{2}{*}{\multicolumn{2}{|c|}{$\begin{array}{l}\text { Renal } \\
\text { insufficiency }\end{array}$}} & \multirow{2}{*}{\multicolumn{2}{|c|}{$\begin{array}{l}\text { Intraoperative } \\
\text { transfusion }\end{array}$}} & \multirow{2}{*}{\multicolumn{2}{|c|}{ Bile leak }} & \multirow{2}{*}{\multicolumn{2}{|c|}{ RO }} \\
\hline & & & & & & & & & & & & & & \\
\hline & NPBD & PBD & NPBD & PBD & NPBD & PBD & NPBD & PBD & NPBD & PBD & NPBD & PBD & NPBD & PBD \\
\hline Cai et al. [21] & 7 & 0 & NR & NR & NR & NR & $N R$ & $N R$ & 57 & 26 & $N R$ & NR & 125 & 42 \\
\hline Kennedy et al. [22] & 4 & 2 & NR & NR & 5 & 0 & NR & $N R$ & NR & NR & NR & NR & $N R$ & NR \\
\hline Zhang et al. [23] & 2 & 13 & 20 & 133 & NR & NR & $N R$ & $N R$ & 13 & 71 & 2 & 30 & 32 & 124 \\
\hline Xiong et al. [24] & 2 & 3 & 27 & 17 & 6 & 3 & 4 & 3 & 24 & 11 & 6 & 3 & $N R$ & NR \\
\hline Farges et al. [25] & 22 & 17 & 128 & 123 & 14 & 5 & NR & NR & 83 & 97 & NR & NR & NR & NR \\
\hline Wronka et al. [26] & 1 & 8 & NR & NR & 1 & 1 & NR & $N R$ & 2 & 0 & NR & NR & $N R$ & NR \\
\hline Ferrero et al. [27] & 3 & 1 & 19 & 21 & 4 & 5 & NR & NR & 17 & 21 & 10 & 7 & NR & NR \\
\hline Su et al. [28] & 0 & 5 & 6 & 17 & NR & NR & NR & $N R$ & NR & NR & NR & NR & $N R$ & $N R$ \\
\hline Li et al. [29] & 5 & 4 & 16 & 20 & NR & NR & NR & NR & NR & NR & NR & NR & 37 & 30 \\
\hline Ribero et al. [30] & 3 & 12 & 24 & 78 & 23 & 6 & NR & NR & NR & NR & 6 & 18 & 32 & 82 \\
\hline Parks et al. [31] & 1 & 1 & 11 & 11 & NR & NR & 1 & 1 & NR & NR & NR & NR & NR & NR \\
\hline Dinant et al. [32] & 2 & 14 & 6 & 56 & NR & NR & $N R$ & $N R$ & $N R$ & $N R$ & $N R$ & NR & $N R$ & $N R$ \\
\hline Hanafy [33] & 3 & 5 & 11 & 27 & 5 & 8 & 0 & 2 & 7 & 15 & 3 & 14 & NR & NR \\
\hline Ercolani et al. [34] & 2 & 25 & $N R$ & NR & NR & NR & $N R$ & $N R$ & NR & $N R$ & NR & NR & $N R$ & $N R$ \\
\hline Gerhards et al. [35] & 3 & 16 & 13 & 59 & NR & NR & NR & NR & NR & NR & NR & NR & NR & NR \\
\hline Chen et al. [36] & 3 & 3 & 14 & 18 & 4 & 2 & 4 & 1 & $N R$ & NR & 0 & 2 & 11 & 13 \\
\hline
\end{tabular}

Continuous data shown represents mean \pm standard deviation or median (minimum-maximum)

$N R$ not report, $P B D$ preoperative biliary drainage group, NPBD non-preoperative biliary drainage group

effects in reducing liver insufficiency. However, the most recent opinion that PBD could not improve the primary outcomes of hCCA patients was suggested by Wronka [26] and Zhang et al. [23], who worried about the complications associated with PBD, including tumor seeding, cholangitis, inflammation, and additional infections. The aim of PBD is to increase liver tolerance to ischemia and reduce intraoperative blood loss [42], and several studies have already demonstrated the significance of PBD [43-45]. However, most of these studies showed benefits without reporting specific characteristics, such as tumor size, concentration of serum bilirubin, liver remnant volume, preoperative complications, and Bismuth classification, so the standard of how and when to use PBD in patients with hCCA is still not clear. In many medical care centers, doctors develop their own suggestion for conducting PBD regarding preoperative TSB concentration: Nimura et al. [46] and Makuuchi et al. [47] suggested PBD at a bilirubin cut-off of $3 \mathrm{mg} / \mathrm{dl}$ to minimize the occurrence of complications, and Hemming et al. [37] preferred $5 \mathrm{mg} / \mathrm{dl}$ of TSB for PBD. However, Su et al. [28] and Ercolani et al. [34] suggested that when the TSB concentration exceeded 10 $\mathrm{mg} / \mathrm{dl}$, the operation should be delayed, and PBD was required. However, whether $\mathrm{PBD}$ with the aim of decreasing the TSB concentration surely improves liver tolerance to perioperative inflammation and ischemia, while considering the increased incidence of tumor seeding, infection, cholangitis, etc., and how to balance the benefits and risks are still unclear.

Regarding the primary outcomes, no statistically significant difference in mortality was found between the PBD and NPBD groups; however, the overall morbidity rate was higher in the PBD group. Interestingly, the overall morbidity rate changed when the studies were divided into the low and high TSB concentration groups. In the low TSB concentration group, the OR was 0.47 , with a statistically significant difference between PBD and NPBD; the OR in the high concentration group reached 0.81 , without a statistically significant difference between PBD and NPBD. At lower TSB concentrations, PBD might increase the risk of infection in the bile duct system [48]; however, as the concentration of TSB increases, liver function is impaired, and the NPBD patient with severe jaundice who underwent surgery may bare more risk for morbidities.

We performed the subgroup analysis based on bilirubin less than $10 \mathrm{mg} / \mathrm{ml}$ and greater than $15 \mathrm{mg} / \mathrm{ml}$ in the preoperative NPBD group; however, it is undeniable that even in the low concentration group, there may be a 
Table 4 Operative data of overall complications

\begin{tabular}{|c|c|c|c|c|c|c|c|c|c|c|c|c|c|c|c|c|}
\hline \multirow{4}{*}{$\begin{array}{l}\text { Author } \\
\\
\mathrm{NPBD}\end{array}$} & \multirow{2}{*}{\multicolumn{2}{|c|}{$\begin{array}{l}\text { Infection } \\
\text { rate }\end{array}$}} & \multirow{2}{*}{\multicolumn{2}{|c|}{ Cholangitis }} & \multirow{2}{*}{\multicolumn{2}{|c|}{$\begin{array}{l}\text { Intra- } \\
\text { abdominal } \\
\text { abscess }\end{array}$}} & \multirow{2}{*}{\multicolumn{2}{|c|}{$\begin{array}{l}\text { Intraoperative } \\
\text { blood loss(ml) }\end{array}$}} & \multirow{2}{*}{\multicolumn{2}{|c|}{ Operation time(min) }} & \multirow{2}{*}{\multicolumn{2}{|c|}{$\begin{array}{l}\text { Second } \\
\text { laparotomy }\end{array}$}} & \multirow[b]{4}{*}{ NPBD } & \multirow[b]{4}{*}{ PBD } & \multirow[b]{4}{*}{ NPBD } & \multirow[b]{4}{*}{ PBD } \\
\hline & & & & & & & & & & & & & & & & \\
\hline & \multirow{2}{*}{\multicolumn{2}{|c|}{ PBD }} & \multirow[b]{2}{*}{ NPBD } & \multirow[b]{2}{*}{ PBD } & \multirow[b]{2}{*}{ NPBD } & \multirow[b]{2}{*}{ PBD } & \multirow{2}{*}{$\begin{array}{l}\text { Anastomotic } \\
\text { leakage } \\
\text { NPBD }\end{array}$} & \multicolumn{2}{|c|}{ Abdominal collection } & \multirow[b]{2}{*}{ PBD } & \multirow[b]{2}{*}{ NPBD } & \multirow[b]{2}{*}{ PBD } & & & & \\
\hline & & & & & & & & PBD & NPBD & & & & & & & \\
\hline $\begin{array}{l}\text { Cai } \\
\text { et al. [21] }\end{array}$ & NR & NR & NR & NR & NR & NR & $\begin{array}{l}1012.5 \pm \\
491.7\end{array}$ & $325 \pm 25$ & $\begin{array}{l}362.5 \pm \\
121.7\end{array}$ & $370 \pm 175$ & 10 & 1 & $\mathrm{NR}$ & NR & NR & NR \\
\hline $\begin{array}{l}\text { Kennedy } \\
\text { et al. [22] }\end{array}$ & NR & NR & NR & NR & $N R$ & NR & NR & NR & NR & $N R$ & NR & NR & $N R$ & NR & NR & NR \\
\hline $\begin{array}{l}\text { Zhang } \\
\text { et al. [23] }\end{array}$ & 6 & 44 & NR & NR & 4 & 36 & $\begin{array}{l}428.25 \pm \\
190.75\end{array}$ & $509 \pm 173$ & NR & NR & NR & NR & 1 & 10 & NR & NR \\
\hline $\begin{array}{l}\text { Xiong } \\
\text { et al. [24] }\end{array}$ & 11 & 13 & 2 & 1 & 2 & 3 & $675 \pm 275$ & $675 \pm 225$ & NR & NR & 3 & 2 & 2 & 1 & 9 & 6 \\
\hline $\begin{array}{l}\text { Farges } \\
\text { et al. [25] }\end{array}$ & 1 & 6 & NR & NR & NR & NR & NR & NR & $330 \mathrm{M}$ & $385 \mathrm{M}$ & NR & NR & NR & NR & NR & NR \\
\hline $\begin{array}{l}\text { Wronka } \\
\text { et al. [26] }\end{array}$ & 9 & 17 & NR & NR & NR & NR & $N R$ & $N R$ & $\begin{array}{l}349.5 \pm \\
124.5\end{array}$ & $312 \pm 117$ & NR & NR & $N R$ & $N R$ & NR & $N R$ \\
\hline $\begin{array}{l}\text { Ferrero } \\
\text { et al. [27] }\end{array}$ & 5 & 12 & 1 & 3 & 2 & 4 & NR & $N R$ & NR & $N R$ & 3 & 4 & $N R$ & $N R$ & 4 & 4 \\
\hline $\begin{array}{l}\text { Su } \\
\text { et al. [28] }\end{array}$ & $N R$ & $N R$ & $N R$ & NR & NR & NR & NR & $N R$ & NR & NR & NR & NR & NR & $N R$ & $N R$ & NR \\
\hline $\begin{array}{l}\text { Li } \\
\text { et al. [29] }\end{array}$ & $N R$ & NR & $N R$ & NR & $N R$ & NR & NR & $N R$ & NR & NR & NR & $N R$ & NR & $N R$ & $N R$ & $N R$ \\
\hline $\begin{array}{l}\text { Ribero } \\
\text { et al. [30] }\end{array}$ & $N R$ & NR & 2 & 40 & NR & NR & $825 \pm 375.0$ & $\begin{array}{l}1164 \pm \\
583.3\end{array}$ & $365 \pm 147.5$ & $\begin{array}{l}551 \pm \\
216.7\end{array}$ & $N R$ & $N R$ & NR & $N R$ & $N R$ & $N R$ \\
\hline $\begin{array}{l}\text { Parks } \\
\text { et al. [31] }\end{array}$ & 3 & 13 & NR & NR & NR & NR & NR & $N R$ & $N R$ & NR & $N R$ & NR & 0 & 1 & NR & NR \\
\hline $\begin{array}{l}\text { Dinant } \\
\text { et al. [32] }\end{array}$ & NR & $N R$ & NR & NR & NR & NR & NR & $N R$ & $N R$ & NR & NR & NR & NR & $N R$ & NR & NR \\
\hline Hanafy [33] & 11 & 27 & $N R$ & NR & $N R$ & $N R$ & $N R$ & $N R$ & $246 \pm 96$ & $348 \pm 138$ & NR & $N R$ & NR & $N R$ & NR & $N R$ \\
\hline $\begin{array}{l}\text { Ercolani } \\
\text { et al. [34] }\end{array}$ & NR & $N R$ & NR & NR & NR & NR & NR & $N R$ & $N R$ & $N R$ & $N R$ & $N R$ & $N R$ & $N R$ & $N R$ & $N R$ \\
\hline $\begin{array}{l}\text { Gerhards } \\
\text { et al. [35] }\end{array}$ & NR & $N R$ & NR & NR & NR & NR & NR & $N R$ & NR & $N R$ & NR & $N R$ & NR & $N R$ & $N R$ & NR \\
\hline $\begin{array}{l}\text { Chen et al. } \\
\text { [36] }\end{array}$ & 4 & 6 & $N R$ & NR & 1 & 1 & $848 \pm 1112$ & $1016 \pm 923$ & $N R$ & $N R$ & $N R$ & NR & NR & NR & 2 & 4 \\
\hline
\end{tabular}

Continuous data shown represents mean \pm standard deviation or median (minimum-maximum)

$N R$ not report, $N P B D$ non-preoperative biliary drainage group, $P B D$ preoperative biliary drainage group

very few patients with more than $15 \mathrm{mg} / \mathrm{ml}$ of high concentration group with lower preoperative TSB concentration. Therefore, the results of the subgroup analysis are very conservative and can only be used as a reference for future related research directions. The difference between the low and high TSB concentration groups most likely indicates that there was a reasonable concentration of TSB for hCCA patients that motivated the decision to perform PBD.

Regarding the secondary outcomes, no statistically significant difference was observed in renal insufficiency, positive surgical margin, intra-abdominal abscess, intraoperative blood loss, operation time, second laparotomy, anastomotic leakage, or abdominal collection between the two groups. In contrast, statistically significant differences were found in hepatic insufficiency, intraoperative transfusion, bile leakage, infection, and cholangitis.

Interestingly, all of the outcomes with a statistically significant difference were consistent with the fact that the NPBD group might have a lower risk of developing infectious complications and requiring intraoperative transfusions. Stents in the bile duct system surely led to a higher infection rate (cholangitis, infection, intra- 
Table 5 Results of meta-analysis comparing NPBD versus PBD for hCCA

\begin{tabular}{|c|c|c|c|c|c|c|c|}
\hline Outcome of interest & No. of studies & No. of patients & OR/WMD & $95 \% \mathrm{Cl}$ & $\boldsymbol{P}$ value & Heterogeneity $\boldsymbol{P}$ value & $I^{2}$ \\
\hline \multicolumn{8}{|l|}{ Primary outcomes } \\
\hline Mortality & 16 & $775 / 1085$ & 0.91 & $0.64,1.30$ & 0.62 & 0.78 & 0 \\
\hline Morbidity & 12 & $553 / 897$ & 0.67 & $0.53,0.85$ & 0.0009 & 0.05 & 0.44 \\
\hline \multicolumn{8}{|l|}{ Secondary outcomes } \\
\hline Hepatic insufficiency & 8 & $430 / 506$ & 3.09 & $1.15,8.31$ & 0.03 & 0.001 & 0.71 \\
\hline Renal insufficiency & 4 & $154 / 129$ & 1.07 & $0.40,2.84$ & 0.89 & 0.33 & 0.13 \\
\hline Intraoperative transfusion & 7 & $546 / 597$ & 0.72 & $0.55,0.94$ & 0.02 & 0.07 & 0.49 \\
\hline Bile leak & 6 & $236 / 433$ & 0.58 & $1.24,1.41$ & 0.04 & 0.04 & $57 \%$ \\
\hline Ro & 5 & $325 / 435$ & 1.36 & $0.93,1.96$ & 0.11 & 0.77 & $0 \%$ \\
\hline Infection & 8 & $414 / 535$ & 0.31 & $0.20,0.47$ & $<0.001$ & 0.16 & $16 \%$ \\
\hline Cholangitis & 3 & $111 / 160$ & 0.18 & $0.007,0.48$ & 0.0007 & 0.15 & $48 \%$ \\
\hline Intra-abdominal abscess & 5 & $147 / 289$ & 0.48 & $0.22,1.32$ & 0.07 & 0.94 & $0 \%$ \\
\hline Intraoperative blood loss & 5 & $315 / 412$ & 32.34 & $-375.83,440.51$ & 0.88 & $<0.001$ & $99 \%$ \\
\hline Operative time & 4 & $235 / 294$ & -63.21 & $-156.16,29.73$ & 0.18 & $<0.001$ & $90 \%$ \\
\hline Secondary laparotomy & 4 & $239 / 117$ & 1.37 & $0.52,3.63$ & 0.53 & 0.47 & $0 \%$ \\
\hline Anastomotic leakage & 3 & $117 / 248$ & 0.55 & $0.15,2.10$ & 0.38 & 0.64 & $0 \%$ \\
\hline Abdominal collection & 3 & $103 / 93$ & 0.9 & $0.40,2.00$ & 0.79 & 0.82 & $0 \%$ \\
\hline
\end{tabular}

The charts filled with gray means valid outcomes $(P<0.05)$

NPBD non-preoperative biliary drainage, $P B D$ preoperative biliary drainage, No. of patients NPBD group/PBD group, OR odds ratio, WMD weighted mean difference, hCCAAA hilar cholangiocarcinoma

abdominal abscess), which may lead to a higher mortality rate, especially when the liver remnant function (FLR) is $<30 \%$ [30].

On the other hand, PBD decreased the hepatic insufficiency rate but to the same degree in both the low and high TSB concentration groups; this was likely caused by the approximately same short duration of high TSB poisoning when the different concentrations of TSB increase according to the degree of obstruction. In the subgroup analysis of bile leakage, similar to that of overall morbidity, within the low TSB concentration group, the NPBD group had a significantly lower risk of bile leakage $(\mathrm{OR}=0.36)$, while in the high TSB concentration group, the OR changed to 0.92 , and the difference lost significance. On the one hand, this confusing conclusion may be caused by the small number of studies (only two studies) in the high TSB concentration group. On the other hand, PBD had already injured the bile duct physically or by infection; thus, when the concentration of TSB increased, the cicatrization after surgery slowed down, and the liver tended to be more susceptible to inflammation $[28,49]$.

Currently, several studies have discussed whether PBD can be performed for hCCA patients with different conditions. Wiggers et al. [49] and Kennedy et al. [22] showed that patients with an FLR $<30 \%$ who underwent PBD could achieve a significantly reduced hepatic insufficiency rate (33\% versus $0 \%$, PBD versus $\mathrm{NPBD})$ and mortality rate $(33 \%$ versus $0 \%$, PBD versus NPBD); however, in patients with an FLR > 30\%, PBD was not superior to NPBD in reducing the hepatic insufficiency rate $(0 \%$ versus $0 \%)$ or mortality rate (0\% versus 9.1\%, NPBD versus PBD). Wronka et al. [26] claimed that PBD was not necessary if hCCA patients had a TSB concentration $>6.2 \mathrm{mg} / \mathrm{dl}$ or $>2.50$ $\mathrm{mg} / \mathrm{dl}$ with preoperative hypoalbuminemia, anemia, or renal dysfunction.

According to our meta-analysis outcomes, PBD was not suitable for patients with hCCA because of the increased risk of bile leakage, cholangitis, infection, intraoperative transfusion, and overall morbidity. However, patients with hCCA and a TSB concentration $>15 \mathrm{mg} /$ $\mathrm{dl}$ may still tend to undergo PBD rather than NPBD due to the lower mortality rate of $\mathrm{PBD}$, especially in patients with a low FLR.

Moreover, clinical hCCA patients may suffer other diseases or conditions (hypoalbuminemia, anemia, renal dysfunction, renal failure, low immune function, etc.), and the benefits and risks of PBD should be evaluated separately to meet a standard. Nevertheless, high-quality retrospective studies and randomized controlled trials (RCTs) are required to draw definitive guidelines for hCCA patients with different conditions. 


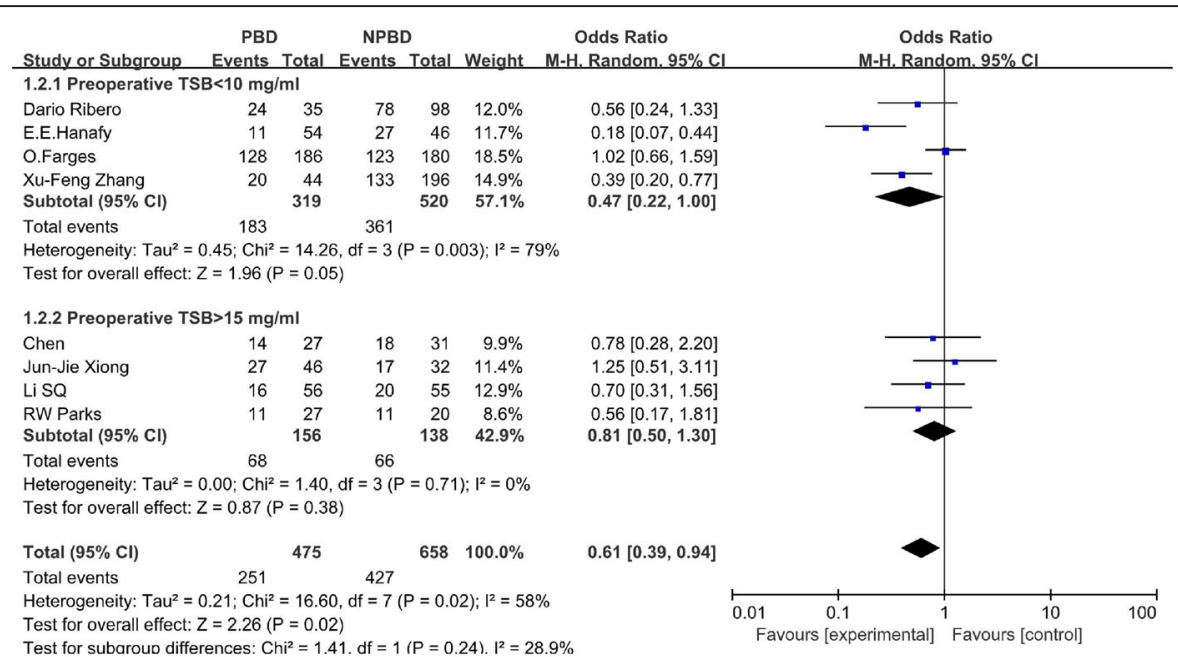

\section{(A) Overall morbidity}

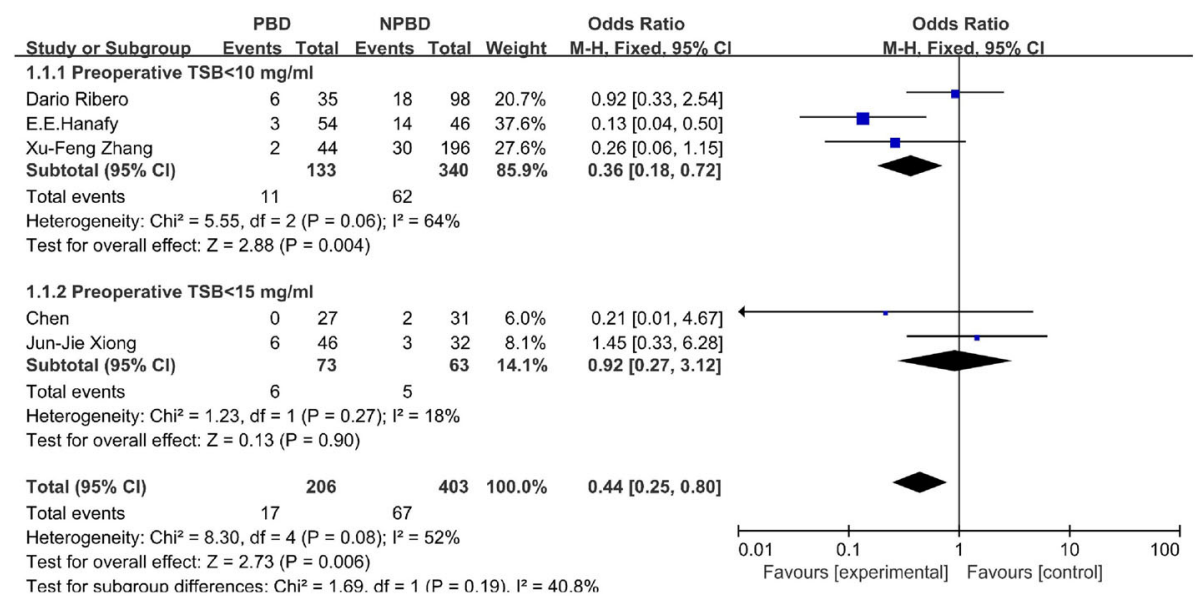

(B) Bile leakage

Fig. 2 Outcomes of the subgroup meta-analysis (according to the NPBD group's preoperative TSB concentration in serum). a Overall morbidity. $\mathbf{b}$ Bile leakage

The meta-analysis still has some limitations. First, the subgroups were roughly divided by the mean TSB of each study $(<10 \mathrm{mg} / \mathrm{dl}$ and $>15 \mathrm{mg} / \mathrm{dl})$. Although we communicated with the authors of the studies to acquire the primary data and widened the TSB difference between the two groups $(<10 \mathrm{mg} / \mathrm{dl}$ and $>15 \mathrm{mg} / \mathrm{dl})$, it was still difficult to obtain fully convincing subgroup outcomes. Second, all of the studies were retrospective studies, which could lead to inevitable selection bias. Third, the studies by Su et al. [28] and Gerhards et al. [35] date back to 1996 and 1999 and perhaps apply different surgical techniques that might influence the results. Fourth, the drainage types included ENBD and
PTBD, which were not mentioned in most of the studies included. Therefore, it is difficult for us to evaluate the bias due to the proportion of ENBD and PTBD procedures performed, although no large differences seemed to be observed.

\section{Conclusion}

The meta-analysis demonstrated that PBD is related to a greater risk of several kinds of infections and morbidities than NPBD, but the ability of PBD to reduce postoperative hepatic insufficiency cannot be ignored. In patients with a high TSB concentration, PBD tends to be a better choice. Nonetheless, these results need to be confirmed 


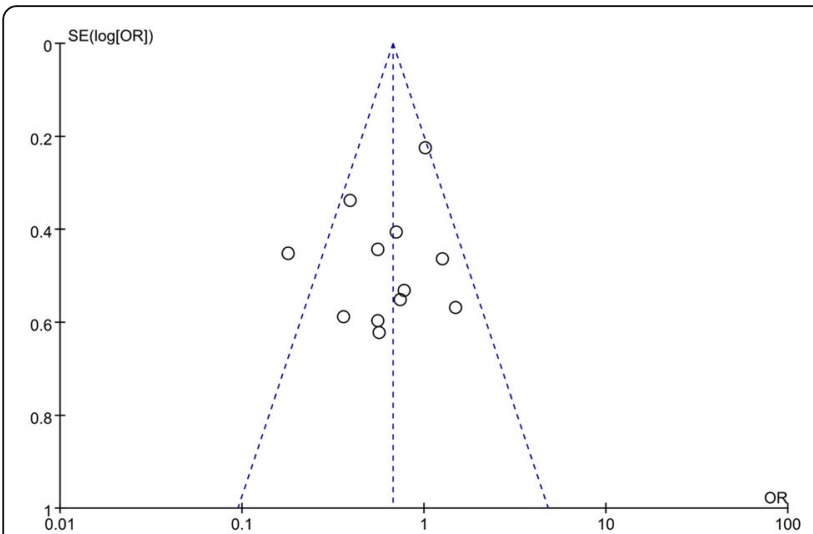

(A)

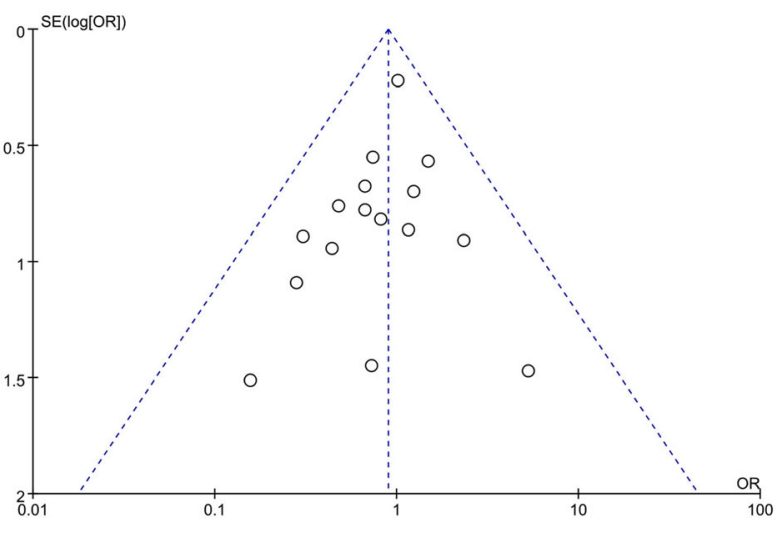

(B)

Fig. 3 Publication bias measured by the funnel plot of 90-day mortality (a) and overall morbidity (b)

in a future prospective randomized trial with large samples to clarify the effects and find a specific TSB concentration for PBD.

\section{Abbreviations}

Cl: Confidence interval; OR: Odds ratio; RR: Relative risk; PBD: Preoperative biliary drainage; NPBP: Non-preoperative biliary drainage; hCCA: Hilar cholangiocarcinoma; TSB: Total serum bilirubin; BMI: Body mass index; FLR: Future liver remnant; PTBD: Percutaneous, transhepatic biliary drainage; ENBD: Endoscopic nasobiliary drainage

\section{Acknowledgements}

None

\section{Authors' contributions}

Study concept and design, Fei Teng and Zhe-yu Chen; acquisition of data, Fei Teng and You-yin Tang; analysis and interpretation, Fei Teng and Junlong Dai; draft the manuscript and preliminary revise, Fei Teng and Yongkun Li; study supervision and final approval, Zhe-yu Chen. All authors read and approved the final manuscript.

\section{Funding}

None. This research did not receive any specific grant from funding agencies in the public, commercial, or not-for-profit sectors.

\section{Availability of data and materials}

The tables and figures supporting the conclusions of this article are included within the article and additional files

\section{Ethics approval and consent to participate}

Not applicable.

\section{Consent for publication}

All authors consented for publication.

\section{Competing interests}

The authors declare that they have no competing interests. The authors report no proprietary or commercial interest in any product mentioned or concept discussed in this article.

Received: 1 April 2020 Accepted: 2 June 2020

Published online: 18 July 2020

\section{References}

1. Khan SA, Davidson BR, Goldin RD, et al. Guidelines for the diagnosis and treatment of cholangiocarcinoma: an update. Gut. 2012;61(12):1657-69.
2. Esnaola NF, Meyer JE, Karachristos A, Maranki JL, Camp ER, Denlinger CS Evaluation and management of intrahepatic and extrahepatic cholangiocarcinoma. Cancer. 2016;122:1349-69.

3. Cai JQ, Cai SW, Cong WM, Chen MS, Chen P, Chen XP, Chen YL, Chen YF, Dai $\mathrm{CL}$, Huang $\mathrm{Q}$, et al. Diagnosis and treatment of cholangiocarcinoma: a consensus from surgical specialists of China. J Huazhong Univ Sci Technol Med Sci. 2014;34:469-75.

4. Poruk KE, Pawlik TM, Weiss MJ. Perioperative management of hilar cholangiocarcinoma. J Gastrointest Surg. 2015;19:1889-99.

5. Nagino M, Kamiya J, Uesaka K, et al. Complications of hepatectomy for hilar cholangiocarcinoma. World J Surg. 2001;25:1277-83.

6. Nanashima A, Abo T, Hamasaki K, Wakata K, Kunizaki M, Tou K, Takeshita H, Hidaka S, Sawai T, Tsuchiya T, et al. Predictors of intraoperative blood loss in patients undergoing hepatectomy. Surg Today. 2013;43:485-93.

7. lacono C, Ruzzenente A, Campagnaro T, Bortolasi L, Valdegamberi A, Guglielmi A. Role of preoperative biliary drainage in jaundiced patients who are candidates for pancreatoduodenectomy orhepatic resection: highlights and drawbacks. Ann Surg. 2013;257:191-204.

8. Nakanishi Y, Tsuchikawa T, Okamura K, Nakamura T, Tamoto E, Noji T, Asano T, Amano T, Shichinohe T, Hirano S. Risk factors for a high Comprehensive Complication Index score after major hepatectomy for biliary cancer: a study of 229 patients at a single institution. HPB (Oxford). 2016;18:735-41.

9. Pitt HA, Gomes AS, Lois JF, Mann LL, Deutsch LS, Longmire WP Jr. Does preoperative percutaneous biliary drainage reduce operative risk or increase hospital cost? Ann Surg. 1985;201:545-53.

10. Kimura N, Young AL, Toyoki Y, Wyatt Jl, Toogood GJ, Hidalgo E, Prasad KR, Kudo D, Ishido K, Hakamada K, et al. Radical operation for hilar cholangiocarcinoma in comparable Eastern and Western centers: outcome analysis and prognostic factors. Surgery. 2017;162:500-14.

11. Celotti A, Solaini L, Montori G, Coccolini F, Tognali D, Baiocchi G. Preoperative biliary drainage in hilar cholangiocarcinoma: systematic review and meta-analysis. Eur J Surg Oncol. 2017;43(9):1628-35.

12. Sterne JA, Hernán MA, Reeves BC, Savovi J, Berkman ND, Viswanathan M, et al. Robins-i: a tool for assessing risk of bias in non-randomised studies of interventions. BMJ. 2016:14919.

13. Hozo SP, Djulbegovic B, Hozo I. Estimating the mean and variance from the median, range, and the size of a sample. BMC Med Res Methodol. 2005;5(1 Supplement):13.

14. Rahbari NN, Garden OJ, Padbury R, Brooke-Smith M, Crawford M, Adam R, et al. Posthepatectomy liver failure: a defifinition and grading by the International Study Group of Liver Surgery (ISGLS). Surgery. 2011;149:713-24.

15. Hochwald SN, Burke EC, Jarnagin WR, Fong Y, Blumgart LH. Association of preoperative biliary stenting with increased postoperative infectious complications in proximal cholangiocarcinoma. Arch Surg. 1999;134: 261-6.

16. Capussotti L, Ferrero A, Viganò L, Sgotto E, Muratore A, Polastri R. Bile leakage and liver resection: where is the risk? Arch Surg. 2006;141:690-4. 
17. Dindo D, Demartines N, Clavien PA. Classifification of surgical complications: a new proposal with evaluation in a cohort of 6336 patients and results of a survey. Ann Surg. 2004;240:205-513.

18. Higgins JPT. Measuring inconsistency in meta-analyses. Bmj. 2003;327(7414): 557-60.

19. Moher D, Liberati A, Tetzlaff J, Altman DG, PRISMA Group. Preferred reporting items for systematic reviews and meta-analyses: the PRISMA statement. PLoS Med. 2009;6(7):e1000097.

20. Figueras J, Codina-Barreras A, Lopez-Ben S, Soriano J, Pardina B, Falgueras L, et al. Major hepatectomies are safe in patients with cholangiocarcinoma and jaundice. Cir Esp. 2009;86(5):296-302.

21. Cai Y, Tang Q, Xiong X, Li F, Ye H, Song P, Cheng N. Preoperative biliary drainage versus direct surgery for perihilar cholangiocarcinoma: a retrospective study at a single center. Biosci Trends. 2017;11(3):319-25.

22. Kennedy TJ, Yopp A, Qin Y, Zhao B, Guo P, Liu F, et al. Role of preoperative biliary drainage of liver remnant prior to extended liver resection for hilar cholangiocarcinoma. HPB (Oxford). 2009;11(5):445-51.

23. Zhang XF, Beal EW, Merath K, Ethun CG, Salem A, Weber SM, et al. Oncologic effects of preoperative biliary drainage in resectable hilar cholangiocarcinoma: percutaneous biliary drainage has no adverse effects on survival. J Surg Oncol. 2018;117(6):1267-77.

24. Xiong JJ, Nunes QM, Huang W, Pathak S, Wei AL, Tan CL, Liu XB. Preoperative biliary drainage in patients with hilar cholangiocarcinoma undergoing major hepatectomy. World J Gastroenterol. 2013;19(46):8731-9.

25. Farges O, Regimbeau JM, Fuks D, Le Treut YP, Cherqui D, Bachellier $P$, et al. Multicentre European study of preoperative biliary drainage for hilar cholangiocarcinoma. Br J Surg. 2013;100(2):274-83.

26. Wronka KM, Grąt M, Stypułkowski J, et al. Relevance of preoperative hyperbilirubinemia in patients undergoing hepatobiliary resection for hilar cholangiocarcinoma. J Clin Med. 2019;8(4):458

27. Ferrero A, Lo Tesoriere R, Vigano L, Caggiano L, Sgotto E, Capussotti L. Preoperative biliary drainage increases infectious complications after hepatectomy for proximal bile duct tumor obstruction. World J Surg. 2009; 33(2):318-25.

28. Su CH, Tsay SH, Wu CC, Shyr YM, King KL, Lee $\mathrm{CH}$, et al. Factors influencing postoperative morbidity, mortality, and survival after resection for hilar cholangiocarcinoma. Ann Surg. 1996;223(4):384-94.

29. Li SQ, Chen D, Liang LJ, Peng BG, Yin XY. The impact of preoperative biliary drainage on surgical morbidity in hilar cholangiocarcinoma patients. Zhonghua Wai Ke Za Zhi. 2009:47(15):1134-7 Chinese. PMID: 20021901.

30. Ribero D, Zimmitti G, Aloia TA, Shindoh J, Fabio F, Amisano M, et al. Preoperative cholangitis and future liver remnant volume determine the risk of liver failure in patients undergoing resection for hilar cholangiocarcinoma. J Am Coll Surg. 2016;223(1):87-97.

31. Parks RW, Currie EJ, Madhavan KK, Garden OJ. Increased bacterobilia associated with preoperative biliary drainage in patients with hilar cholangiocarcinoma. HPB. 2000;2(4):375-81.

32. Dinant S, Gerhards MF, Rauws EA, Busch OR, Gouma DJ, van Gulik TM. Improved outcome of resection of hilar cholangiocarcinoma (Klatskin tumor). Ann Surg Oncol. 2006;13(6):872-80.

33. El-Hanafy E. Pre-operative biliary drainage in hilar cholangiocarcinoma, benefits and risks, single center experience. Hepatogastroenterology. 2010; 57(99-100):414-9.

34. Ercolani G, Zanello M, Grazi GL, Cescon M, Ravaioli M, Del Gaudio M, et al. Changes in the surgical approach to hilar cholangiocarcinoma during an 18-year period in a Western single center. J Hepatobiliary Pancreat Sci. 2010; 17(3):329-37.

35. Gerhards MF, van Gulik TM, de Wit LT, Obertop H, Gouma DJ. Evaluation of morbidity and mortality after resection for hilar cholangiocarcinoma--a single center experience. Surgery. 2000;127(4):395-404.

36. Chen D, Peng BG, Shao-Qiang LI. Effect of preoperative biliary drainage on hilar cholangiocarcinoma in patients underwent resection operation. Chin J Pract Surg. 2007; Doi:1005-2208(2007)06-0805-04.

37. Hemming AW, Reed Al, Fujita S, Foley DP, Howard RJ. Surgical management of hilar cholangiocarcinoma. Ann Surg. 2005;241:693-9 discussion 693-699.

38. Kloek JJ, Marsman HA, van Vliet AK, et al. Biliary drainage attenuates postischemic reperfusion injury in the cholestatic rat liver. Surgery. 2008;144: 22-31.

39. lacono C, Ruzzenente A, Campagnaro T, et al. Role of preoperative biliary drainage in jaundiced patients who are candidates for pancreatoduodenectomy or hepatic resection: highlights and drawbacks. Ann Surg. 2013:257:191-204.

40. Rerknimitr R, Angsuwatcharakon P, Ratanachu-ek T, et al. Asia-Pacific consensus recommendations for endoscopic and interventional management of hilar cholangiocarcinoma. J Gastroenterol Hepatol. 2013;28: 593-607.

41. Olthof PB, Coelen RJ, Wiggers JK, et al. External biliary drainage following major liver resection for perihilar cholangiocarcinoma: impact on development of liver failure and biliary leakage. HPB (Oxford). 2016;18:348-53.

42. Vauthey $\mathrm{JN}$, Blumgart $\mathrm{LH}$. Recent advances in the management of cholangiocarcinomas. Semin Liver Dis. 1994;14:109-14.

43. McPherson GA, Benjamin IS, Hodgson HJ, Bowley NB, Allison DJ, Blumgart $\mathrm{LH}$. Pre-operative percutaneous transhepatic biliary drainage: the results of a controlled trial. Br J Surg. 1984;71(5):371-5.

44. Hatfifield AR, Tobias $R$, Terblanche J, et al. Preoperative external biliary drainage in obstructive jaundice. A prospective controlled clinical trial. Lancet. 1982;2:896-9.

45. Lai EC, Mok FP, Fan ST, et al. Preoperative endoscopic drainage for malignant obstructive jaundice. Br J Surg. 1994;81:1195-8.

46. Nimura Y, Hayakawa N, Kamiya J, Kondo S, Shionoya S. Hepatic segmentectomy with caudate lobe resection for bile duct carcinoma of the hepatic hilus. World J Surg. 1990;14:535-43 discussion 544.

47. Makuuchi M, Thai BL, Takayasu K, et al. Preoperative portal embolization to increase safety of major hepatectomy for hilar bile duct carcinoma: a preliminary report. Surgery. 1990;107(5):521-7.

48. Lai EC, Chu KM, Lo CY, et al. Surgery for malignant obstructive jaundice: analysis of mortality. Surgery. 1992;112(5):891-6.

49. Wiggers JK, Groot Koerkamp B, Cieslak KP, et al. Postoperative mortality after liver resection for perihilar cholangiocarcinoma: development of a risk score and importance of biliary drainage of the future liver remnant. J Am Coll Surg. 2016;223(2):321 331.e1.

\section{Publisher's Note}

Springer Nature remains neutral with regard to jurisdictional claims in published maps and institutional affiliations.
Ready to submit your research? Choose BMC and benefit from:

- fast, convenient online submission

- thorough peer review by experienced researchers in your field

- rapid publication on acceptance

- support for research data, including large and complex data types

- gold Open Access which fosters wider collaboration and increased citations

- maximum visibility for your research: over $100 \mathrm{M}$ website views per year

At BMC, research is always in progress.

Learn more biomedcentral.com/submission 